\title{
Implementation of Five Key Process Areas to Improve the Requirement Engineering Process
}

\author{
Sathiya \\ Research Scholar \\ SCSVMV University
}

\author{
K. Mythili \\ Assistant Professor \\ SCSVMV University
}

\begin{abstract}
Requirement engineering is the most powerful phase of software development process. It targets to collect good requirements from stakeholders in the right way. Requirements engineering is the initial phase of software engineering process in which user requirements are gathered, understood, and state clearly for developing quality software products. The current software process improvement standards have no special section mentioned to requirements engineering process and they widely treat it as a single activity in the overall development process. This research displays that in order to produce quality software greater attention must be given to the improvement of RE process. In this research paper five key process areas have been identified in order to improve the RE process. This research project aims to display that quality requirements will come after when the RE process supports these five KPAs. To implement these KPAs, a requirement elicitation, analysis and validation method (REAVM) is proposed. A framework has been developed in order to evaluate the REAVM.
\end{abstract}

\section{Keywords}

Requirements Engineering Process, Key Process Areas, REAVM, Requirement Engineering, Requirement Analysis, Requirement Elicitation, Requirement Development, Requirement Management.

\section{INTRODUCTION}

Inaccurate, insufficient, or misunderstood requirements are the most common reasons of poor quality, cost overruns and late delivery of software systems. Requirements problems are broadly accepted to shorten the quality of software and to impulse on the effectiveness of the software development process. In spite of the importance of requirements engineering, little work has been done on developing ways to improve requirements process. Existing standards for SPI, and ISO 9000 series standards do not address the requirements engineering sufficiently.

Many software projects have failed because they hold a poor set of requirements. No software process can hold delivery times, costs and product quality under control if the requirements are poorly defined. In order to produce software, which closely matches the needs of an organization, an application domain and the stakeholders, great attention must be given to the RE process. The RE process acts an important role in the software development process.

Requirements engineering is an important process of the software life-cycle. Some examples of fairly common problems with the RE process are as follows:

\footnotetext{
- Business needs are not considered.

- Lack of requirements management.

- Lack of defined responsibilities.
}

\author{
- Undefined requirements process. \\ - Lack of stakeholder involvement. \\ - The requirements do not throw back the real needs \\ of the customers. \\ - Requirements growth. \\ - Stakeholder's communication problems. \\ - Requirements are not uniform and/or not finished. \\ - It is costly to make changes to requirements after \\ they have been agreed.
}

Requirement engineering is an orderly approach through which the software engineer collects requirements from different sources and implements them into the software development processes.

Requirements engineering hold a set of activities for discerning, analyzing, documenting, validating and maintaining a set of requirements for a system. Requirement development conceals activities related to discovering, analyzing, documenting and validating requirements whereas requirement management includes activities related to traceability and change management of requirements. Requirements verification includes such activities that make sure that the product of a system development process meets its technical specifications. Requirements validation includes some activities that make permanent that the behavior of a developed system meets its user needs. Requirement engineering is a very important activity, which can make a show of the entire activity of software development project.

\section{RELATED WORK}

Sommerville have published the RE process maturity model which has been derived from the existing standards and has three levels, i.e. Level 1 - Initial, Level 2 - repeatable and Level 3 - Defined. This model can be used to evaluate current $\mathrm{RE}$ process and it provides a template for requirements engineering practice assessment. This model does not supply any common methodology for the improvement of the RE process. However, it also does not provide KPA's like CMM but rather it organizes different requirements practices with various deliverables in the RE process.

Dhirendra Pandey, A.K.Ramani and U.Suman denoted that Requirements Engineering for software development process is an intricate exercise that considers product demands from enormous number of outlooks, functions, responsibilities and objectives. For that reason, it becomes necessary to apply requirement engineering practices in every phase of software development process. We propose an effective requirements engineering process model to create quality requirements. Requirement management and planning phase is executed independently for a powerful management of requirements. 
The effective implementation of proposed requirement engineering process can have a good effect on the production of quality software product.

Mahmood Khan Niazi stated that the current software process improvement (SPI) standards have no special section referring to requirements engineering process and they widely treat it as a single activity in the overall development process. The RE process plays an important role in the software development process and its importance demands that it be recognized as a process in its own right and not simply as a phase of the software life-cycle. Research displays that in order to create quality software greater attention must be given to the improvement of RE process.

Lea Reinikainen says that Requirements Engineering is an important phase in software development, because misidentification of requirements is one of the most important sources of customer dissatisfaction with delivered systems. Software engineers use a number of methods and techniques to draw out requirements. The field of all possible requirements elicitation techniques is vast. The objective of this paper was to improve the requirements elicitation process. An improved requirements elicitation process was developed on the basis of evaluation on different requirement elicitation techniques.

\section{TOOLS AND TECHNIQUES 3.1 Improving the Requirement Engineering process}

The major objective of this research project is to improve the $\mathrm{RE}$ process. Because if the RE process is improved, quality requirements can be achieved and the real needs of the stakeholders can be reflected. This research project points out to display that quality requirements will follow when the RE process supports the following:

\subsection{Five Key Process Areas (KPA)}

\subsubsection{To sustain a goal-based approach in the RE} process

Goals are the high level objectives of the business, organization or system which supply a framework for the desired system. Goals are useful for organizing and justifying requirements. Goals have been familiarized into requirements engineering for a different kinds of reasons, i.e. requirements acquisition, relating requirements to the organizational and business context, making clear requirements, documenting requirements and business relations with conflicts, helping the management of change and driving the initial design. Goals set a memorandum by which requirements are discerned, analyzed and documented. Normally, it is hard for the stakeholders to completely understand the requirements of the organization or application domain but with clear goals a good understanding can be gained. By concentrating on goals initially alternative of wide requirements, analysts make able stakeholders to transmit using a language based on ideas with which they are both convenient and familiar.

\subsubsection{To maintain the incremental and cyclical behaviors in the RE process}

Some studies firmly suggested that the requirements process is cyclical. "Potts" have proposed a cyclical model, called the Inquiry Cycle that consists of three iteratively repeated activities: expression, discussion and obligation. "Boehm" have proposed a requirements process model based on its spiral model of software development, which demonstrate stakeholders "win" conditions and includes steps in order to stimulate identification and negotiation of requirements tradeoffs. "Sommerville" have also proposed a spiral model that consists of three iterative activities: requirements elicitation, requirements analysis and validation and requirements negotiation. The incremental behavior is considered as the most realistic approach to software development for largescale systems. Incremental behavior uses an evolutionary approach to development and holds the systematic and the "development in steps" approach of the traditional project life cycle. Using this conduct the functionality of the system is produced and delivered to the customers in small increments which keep away from the "Big Bang" effect.

\subsubsection{To put courage in stakeholders involvement in the RE process}

In most case, the concerned stakeholders are not involved in the RE process and their real needs are not considered in the system. Involving the stakeholders in the development process can reduce their cowardice, for example, that the development of a software system will effect in loss of jobs. It is also that can be done that, if a new system is to located in an organization without consulting the stakeholders, those the stakeholders would be affected by the system, then they may be affected that a new system is needless and therefore they contribute to not co-operate in its specification. Stakeholders involvement in the RE process is one of the most important factors that tend to the accomplishment of the project. With the stakeholders involvement less rework of the documentation items is needed, real requirements can be collected and political conflicts are reduced.

\subsubsection{To support the management of RE process}

During the RE process new requirements appear and existing requirements change at all stages of the system development process. It is frequently the case that more than $50 \%$ of system requirements will be changed before it is put into service. The $\mathrm{RE}$ process is a learning process and ideas produced at one point may change at another point. This development by stages of requirements throughout the whole software development life cycle has to be conducted in order to make sure high quality specifications. The management includes issues such as information storage, organization, traceability and documentation. Requirements management may look like an overhead in the RE process, but it is usually rewarded by better customer satisfaction and lower system development costs.

\subsubsection{To define a planning phase for the RE} process

Powerful management of a software project depends on thoroughly planning the project. Normally, the RE process is begin without any planning and the requirements engineers inevitably wish to start very rapidly. The RE process will be an unproductive exercises if started accidentally and without planning. Particular attention should be paid to the planning of the RE process.

\subsection{Implementation of five key process areas}

In order to implement the five KPA's a "Requirements Elicitation, Analysis and Validation Method (REAVM)" has been developed. This method has been derived from the cyclical and incremental models and has an iterative and 
feedback nature. The motive for the development of a method is that a method is a systematic way of working by which one can reach a desired result. A method supplies a prescription for how to perform a collection of activities, concentrating on how a related set of techniques can be incorporated and supplying guidance on their use. All the identified KPA's are integrated in this method. The development of a method is heavily dependent on a thorough definition of its processes, roles, activities and interactions. More-over, recent trends focusing on process technology have confirmed that a quality product can only be the result of a quality process. Thus, a process oriented approach to method definition has been chosen as the basis for this research project. REAVM is divided into five major phases and each phase is an organized set of activities which transforms inputs to outputs. Each phase takes an input, adds value to it and provides an output. The output of a phase is used as an input for the next phase and so on.

\subsection{The structure of REAVM}

REAVM is derived from the cyclical and incremental models and is divided into five phases:

$\begin{array}{ll}\text { - } & \text { Planning. } \\ \text { - } & \text { Requirements elicitation. } \\ \text { - } & \text { Requirements analysis. } \\ \text { - } & \text { Requirements agreement. }\end{array}$

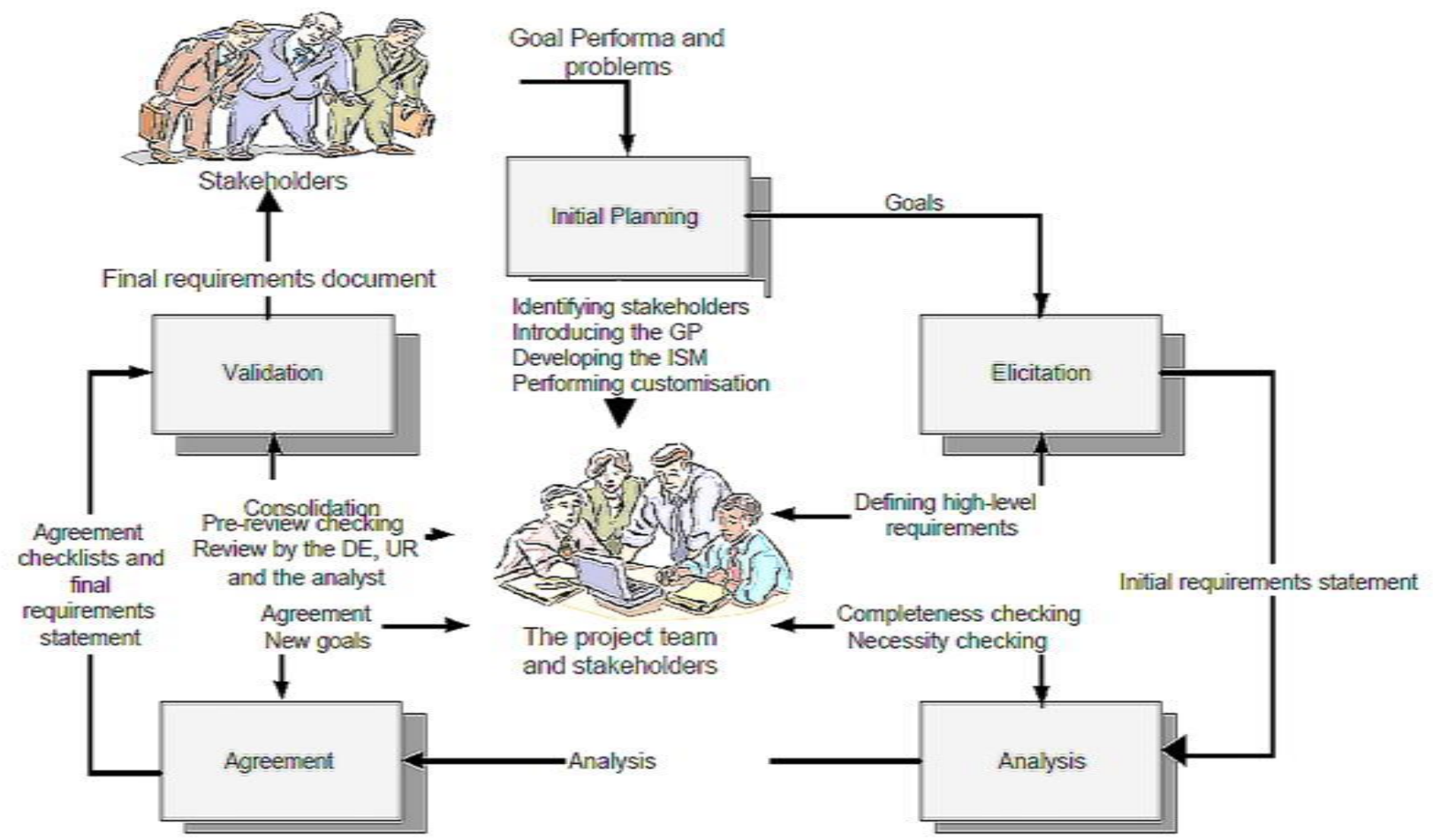

Figure 1: The structure of REAVM

The above figure illustrates proposed cyclical model that has been abstracted from different studies. It is cyclical in that requirements become obvious from consecutive iterations in the context of the requirements which appear from previous iterations. Hence requirements which come into view in the later iteration may limit requirements which emerge in the previous iteration.

In this cyclical model, five activities are repeated each iteration of the REAVM cycle. This model works at two levels: In the first level, only one goal is considered for REAVM cycle. After first cycle of REAVM, if enough information is not gathered or some conflicts are still not determined then the same goal is re-considered for the second cycle of REAVM and so on. Through this cyclical behavior requirements will become easily seen and it is possible that the requirements produced in the later iteration may limit requirements generated in the previous iteration. In the second level, after the perfection of first goal then the second goal is considered for REAVM cycle and as mentioned earlier requirements which appear in the iteration of second goal may limit requirements which appeared in the iteration of first goal. Hence requirements draw out in each cycle of REAVM are validated with the previous elicited requirements for consistency, completeness and feasibility. 


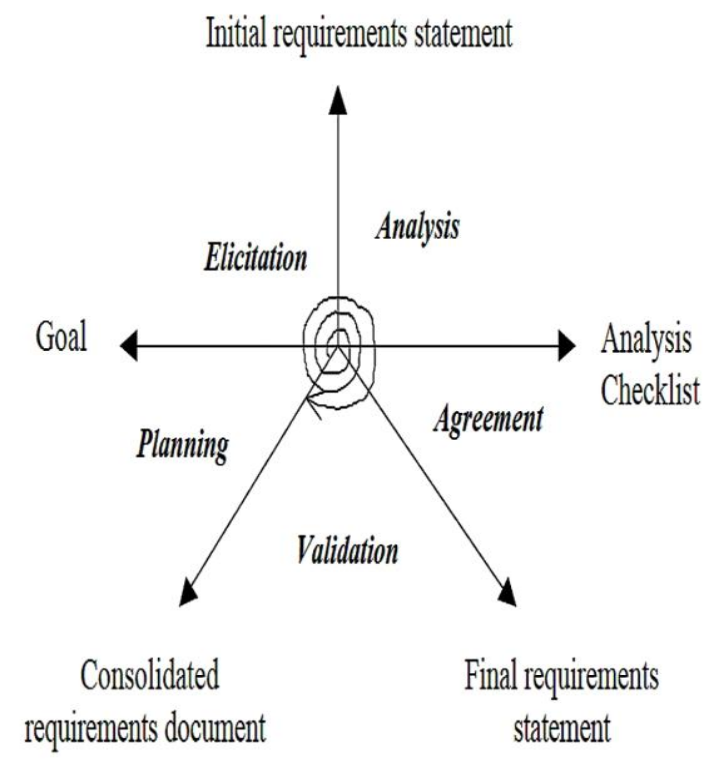

Figure 2: Cyclic behavior of REAVM

\section{Incremental behavior of REAVM}

This method assumes that the requirements for large systems are incrementally collected, analyzed and validated using multiple builds as shown in the following figure. The initial planning for build-1 is carried out at the commencement of the project. Further planning is carried out as and when needed, as new goals can appear during separate phases. The next three phases, elicitation, analysis, and agreement are performed once for each build. The last phase, validation is performed after each build. This shows the incremental behavior.

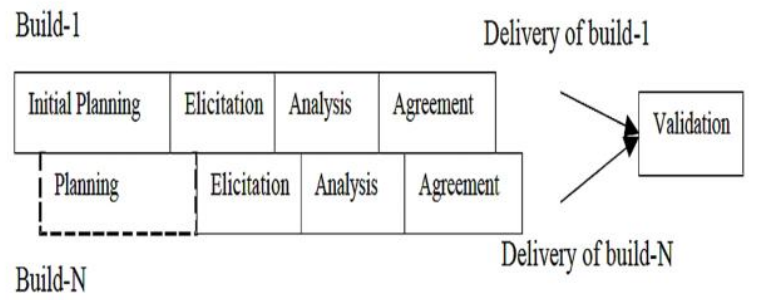

Figure 3: Incremental behavior of REAVM

\subsubsection{Planning phase}

This is the first phase of REAVM. The aim of this phase is to prepare some planning for the subsequent phases of REAVM. Four types of stakeholders are identified during planning phase, i.e. the executive sponsor, the analyst, the domain experts $[\mathrm{DE}]$ and the user representatives [UR]. The executive sponsor is the manager or executive who is having responsibility for making executive level opinions and obligations. The analyst is having responsibility for different tasks of REAVM. A domain expert is a person who can supply detailed information on an illiberal, well-defined topic. They have the best available scene of a remarkable domain area.

In order to implement goal-based approach, the goal Performa [GP] is familiarized in REAVM to demonstrate the goal and the flow of that goal from each DE and UR. According to simple diagrams, supplemented by descriptions of the system realities are the suitable starting points for describing system contexts. The GP is build up by assuming that the stakeholders have goals in their minds. One example of the GP is shown in Table 1.

Goal Number $\quad: \quad 6$
Goal Name
Description of Goal : Modification of application for license
(production) ALI
Source of Goal : Central Excise Circles, Domain
Expert (ALI) and User Rep. (ALI)
Function of Goal : The activity for collection of
excise duty starts with an application for commencement of
business, production. The application is lodged with the
circle office on prescribed forms ALI for issuing of license.
Problems : The current ALI form not contain
all the required information

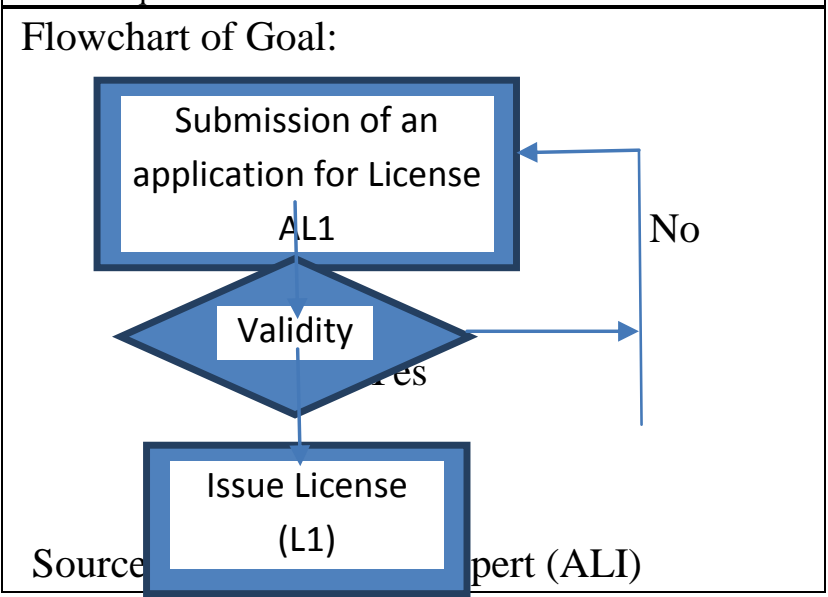

Table 1: Goal Performa

In the planning phase different essential tasks are carried out by the analyst, i.e. goal identification, goal prioritization, team organization, assigning of responsibilities and preparation of different materials to be used in different phases of REAVM. Teams are arranged according to the goals. Responsibilities are assigned to different stakeholders for elicitation, analysis, agreement and validation phases. Also some materials are provided which can be used during different phases of REAVM.

\subsubsection{Requirements elicitation phase}

The goals which are generated in the initial planning phase are taken as input. The goal which has the largest priority number is considered first for elicitation and so on. The following steps are carried out in this phase:

- Defining high-level requirements.

- Resolving the scope of the requirements.

- Generation of initial requirements statements.

The analyst stimulates the group discussions that elicit the requirements. In order to implement Stakeholders involvement KPA's, the participants are induced to bring different thoughts and opinions about different problems. Participants are also invited to declare their viewpoints about any of the problems. Different questions are required from each DE and UR using the lists of questions prepared in the initial planning phase. Every participant is permitted to present his viewpoint, if needed. Through carefully make easy 
discussions, the ideas and views about the above topics are presented, examined and refined, so that by the end of the elicitation phase everyone is in agreement. If necessary, interviews can be guided with those who are not to take part in the meeting but they have some relation with the goal under consideration.

At the end of the elicitation phase an initial requirements statement is produced for each goal by the analyst and is given to each DE and UR for analysis and discussion.

$\begin{array}{ll}\text { Goal Number: } & 6 \\ \text { Goal Name } & : \quad \text { Modification of ALI }\end{array}$

Description of Goal: Modification of application for license (production) ALI

Source of Goal: $\quad$ Central Excise Circles, Domain Expert (ALI) and User Rep. (ALI)

Function of Goal: The activity for collection of excise duty starts with an application for commencement of business, production. The application is lodged with the circle office on prescribed forms ALI for issuing of license.

Problems: The current ALI form not contain all the required information

Elicited Requirements:

Requirement 1: The ALI form shall be updated to contain all the required information and the new ALI form shall contain the following production unit information:

Circle name, unit national tax number, unit license number, unit name, unit address, unit

telephone number, unit fax number, unit paid up capital, unit license expiry date.

Requirement 2: Details regarding ownership:

2.1 If an individual is the owner of the unit, then his national tax number, NIC number, name, address, telephone number, fax number, and email address shall be provided.

2.2 If the unit is registered firm then the registration number, date of registration, expiry date and issuance authority for the firm shall be provided.

(.............

Requirement $\mathrm{n}$

\section{Table 2: Initial requirements statement}

\subsubsection{Requirements analysis phase}

The goal of this phase is to discover problems in the initial requirements statements produced in the requirements elicitation phase of REAVM. In the requirements analysis phase the following types of checking is carried out using the analysis checklist:

\section{- $\quad$ Completeness checking. \\ - Necessity checking. \\ - General comments.}

Stakeholders analyzed the requirements for completeness. Completeness means that no requirements that are needed have been omitted, i.e. whether the elicited requirements have protected all of the needs and objectives of the organization, application domain and stakeholders. An initial requirements statement can be considered as complete when all of its parts are present and no delayed decision or no 'to be defined' statements, still exist. In completeness checking the incomplete requirements are pinpointed.

At the foot of the analysis checklist the stakeholders have to provide general comments about the initial requirements statement.

\subsubsection{Requirements agreement phase}

The agreement phase is the process of commenting the issues or problems pointed out by the DE's and UR's in the requirements analysis phase of REAVM and finding some agreement with which all of the stakeholders can live. Solutions are identified and issues are determined to the satisfaction of the parties included. Generally, this will include deletion of some requirements and making changes to some of the requirements in order to improve them. In many cases, it is possible that some questions may be raised which cannot be answered and for which the stakeholders may not agree with the proposed solutions. This means that the information available for the agreement is inadequate. In such cases, the undetermined issues are forwarded again to another round of REAVM.

This phase is concluded by reviewing with the participants the information collected and the decisions made. At the end of this phase, the final requirements statements and the agreement checklists are generated and forwarded to the validation phase for validation and discussion.

Goal Number: Goal Name: Description of Goal:

Final Elicited Requirements:

Requirement 1:

Requirement $\mathrm{n}$ :

\section{Table 3: Final requirements statement}

\subsubsection{Requirements validation phase}

This is the final phase of REAVM. In REAVM, only one goal is considered for elicitation, analysis and agreement at any one time. It is therefore possible that some infeasibility, inconsistency and incompleteness may exist when all the goals are united into one document. It is also possible that some previous requirements may change because the customers can change their minds or even the environment of the system laws or regulations might change. Therefore, the objective of this phase is to check and remove such infeasibility, inconsistency or incompleteness and to qualify the changed requirements to the new requirements. The final requirements statements produced in the agreement phase of REAVM are consolidated into one requirements document after each cycle or build. Each goal has its own serial number, so using this serial number all the final requirements statements are consolidated sequentially into one document.

The DE's and UR's read and analyze the requirements document and look for different problems e.g., changed requirements, inconsistencies, incompleteness and infeasibility. If some requirements are not finished then for those particular requirements the elicitation, analysis, agreement and validation phases can be carried out again. If some requirements are inconsistent then meetings are held between the stakeholders whose requirements are inconsistent in order to reach agreement and to take away these inconsistencies. If some requirements are infeasible, then 
those requirements are changed or removed and if some requirements are modified then those requirements are changed according to new requirements.

\subsection{Evaluation of REAVM}

REAVM has been estimated using the evaluation framework developed from the research literature. This framework has been developed using five main criteria's. First, it should be geared to the RE process. Second, it should clearly differentiate between the stages of RE process. Third, its dimensions should be well used and well known. Fourth, it should have basic objective to improve or evaluate the RE process. Fifth, it should incorporate the objectives of this research project. This framework has 4 components and 28 dimensions and provides a very practical framework with which to estimate the REAVM. Against each dimension, one of the following assessments is made.

- Fulfills criteria. This means that the dimension fully describes the process or practice that has a documented standard in REAVM.

- Partially fulfills criteria. This means that the dimension partially describes the process or practice.

- Do not Fulfills criteria. This means that the dimension does not describe the process or practice.

Out of 28 dimensions, 18 fulfilled the criteria, 3 partially fulfilled the criteria and 7 did not fulfill the criteria. REAVM fulfilled $64 \%$ of the criteria, partially fulfilled $11 \%$ of the criteria and did not fulfill $25 \%$ of the criteria.

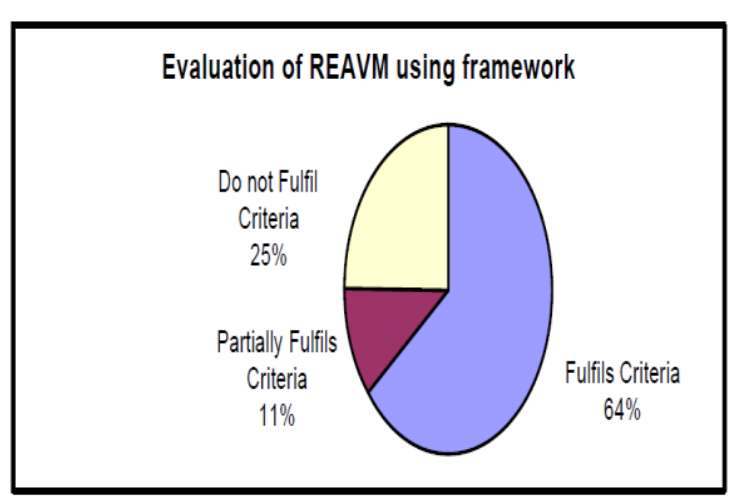

Figure 4: Evaluation of REAVM

The assessment criteria has been adopted from the above model where the requirements process is considered matured if it gets more than 55 points [50\%] in the basic guidelines. If roughly $50 \%$ is considered an average success criterion then satisfactory results have been achieved.

\section{RESULT \& DISCUSSION}

This paper examined issues relating to about the improvement of requirement engineering process in requirements engineering. This research project aims to show that quality requirements will follow when the RE process supports these five KPA's. To implement these KPA's, a requirement elicitation, analysis and validation method [REAVM] is proposed. A framework has been developed from the research literature in order to evaluate the REAVM.

The proposed requirement engineering process is more powerful to create quality requirements. The proposed requirement engineering model can be used in larger software development process. It presents the new intuition of requirement management and planning which can control the changing requirement. It can help to get back and change the requirement, whenever requirements are modified in any phase of software development. Software developer can easily provide the need and change requirements using requirement management. Software developer can plan the software product, which will be having the power to avoid and control changing requirements.

The first stage in process improvement was to identify the areas where a company is weakest as these are where improvements are most likely to be cost effective. We therefore identified the need for more detailed information on the use of good practice in different areas of the requirements engineering process than was available from the maturity and area or strength matrices.

We decided to target an area for improvement if fewer than $50 \%$ of the Basic guidelines in an area were used. This was based on the assumption that familiarizing new guidelines probably needed more support than standardizing guidelines that were already used.

\section{CONCLUSION}

For the improvement of RE process, five KPA's were considered and it was believed that if these KPA's have been considered then the RE process will be improved. As a whole, REAVM performed fairly well. It is believed that the KPA's selected for the improvement of RE process are the best group in order to raise the capacity of the RE process but the way these KPA's are structured into REAVM require further refinement and improvement. It is also believed that REAVM can be further improved through large scale case studies.

The proposed requirement engineering process is more powerful to create quality requirements. The proposed model introduces all important and hidden views of requirement engineering process whereas the existing requirement engineering process models are not able to transmit their phases with software development process in the right manner. We narrate all the important aspects of requirement engineering process to software development process in order to find out good requirements from different sources that can be implemented into software development process for producing quality software products.

We have also narrate requirement management and planning phase to the software development phases in our model because requirements can modify over the time and during software development process this can give bad results. Therefore, it is necessary to control continuously modifying requirements through requirement management and planning. The proposed requirement engineering model can be used in larger software development process to modify the requirements continuously.

Using this model, organization can permit users for any further modification in requirements during software development process. The SRS can be changed accordingly and these modified requirements can be re-implemented in software development. 


\section{REFERENCES}

[1] Boehm B.w., Bose p., Horowtiz E. and Lee M-J (1994) Software requirement as negotiated win conditions, First International Conference on Requirement Engineering.

[2] Boehm B.W. (1998) A Sprial Model of Software Engineering Development and Enhancement, IEEE computer.

[3] Kamsties Erik, Hormann Klasu and Schlich Maud (1998) Requirements Engineering in small and Medium Enterprises, Requirement Engineering.

[4] Kotonya Gerald and Sommerville Ian (1998) Requirement Engineering Processes and Techniques, John Wiley \& Sons.

[5] Lamsweerde Axel van (2001) Goal-oriented Requirements Engineering: A Guided Tour, Proceedings of the $5^{\text {th }}$ International Symposium on Requirements Engineering, IEEE CS Press.
[6] Niazi Mahmood (2000) A Method to Improve the Requirements Engineering Process, Fifth Australian Workshop on Requirements Engineering.

[7] Pressman S.Roger (1997) Software Engineering - A Practitioner's Approach, the McGraw - Hill Companies, Inc.

[8] Sommerville I., Sawyer P. and Viller s. (1997) Requirement Process Improvement Through the Phased Introduction of Good Practice, Software Process Improvement and Practice.

[9] Sommerville I. (1996) Software Engineering Fifth Edition, Addison - Wesely.

[10] Vliet Van Hans (1993) Software Engineering Principles and Practice, John Wiley and sons.

[11] Wieringa R. J. (1996) Requirement Engineering Frameworks for Understanding, John Wiley \& Sons.

[12] P. Jalote, An Integrated Approach to Software Engineering, $3^{\text {rd }}$ edition, Narosa Publishing house, India, 2005. 